\title{
Off-shell effects in heavy particle production
}

\author{
G.F.Bertsch ${ }^{a}$ and P. Danielewicz ${ }^{b}$ \\ ${ }^{a}$ Physics Department and Institute for Nuclear Theory \\ University of Washington, Seattle, WA 98195 \\ and \\ ${ }^{b}$ Department of Physics and Astronomy and National Superconducting Cyclotron Laboratory \\ Michigan State University, East Lansing, MI 48824
}

\begin{abstract}
Off-shell propagation of nucleons is neglected in one-body transport models of heavy-ion collisions, but it could be significant in processes that are limited by phase space, such as the threshold production of heavy particles. We estimate the relative magnitude of off-shell production to on-shell production of the $\mathrm{N}^{*}(1535)$ resonance in heavy ion collisions. In the region where the onshell production is dominated by a two-step mechanism with an intermediate $\Delta$, we find that the contribution of off-shell scattering between projectile and target nucleons is indeed small. Beyond the latter contribution, however, correlations in the initial wave function produce off-shell contributions which can exceed those of the on-shell $\Delta$ mechanism.
\end{abstract}

PACS numbers: 24.10.Cn,25.75.+r

Typeset using REVTEX 
One-body transport theory is widely applied to interpret heavy-ion reactions. In the usual application of the theory, such as with the Boltzmann-Uehling-Uhlenbeck (BUU) equation [1], one assumes that particles propagate classically between collisions. A more complete theory would allow off-shell propagation of particles; the validity of BUU and similar theories depends on these off-shell effects being small.

Recently the claim has been advanced that near-threshold heavy-particle production in nucleus-nucleus collisions is mediated by intermediate production of delta resonances [2] 0 . The conclusion was based on studies within the BUU theory, and thus depends on off-shell propagation being insignificant.

Our objective in this letter is to estimate off-shell effects for such processes. We will make several drastic simplifying assumptions, in order to derive simple formulas. First, we shall consider the stationary rate of production from the initial distribution of nucleons in the colliding nuclei. We also neglect the Fermi momentum of the nucleons, assuming that in zeroth order they are in momentum eigenstates at the beam and target velocities. The sensitivity to off-shell effects should be highest below the energy threshold for the heavy particle in a two-body collision. At such energies the lowest graph that contributes involves three particles, as shown in Fig. 1. In the graph, the particle labeled by $k_{3}$ is a baryon resonance such as the $N^{*}(1535)$. The intermediate particle, labeled by $\mathrm{R}$, could be a delta resonance or a nucleon. The kinematics is such that a nucleon always propagates off its energy shell; the delta may be on shell or not depending on the momenta in the final state.

The two interactions will be much simplified; at the moment we denote the two matrix elements as $M_{1}$ and $M_{2}$. Then the amplitude to go from an initial state with momenta $\left(p_{1}, p_{2}, p_{3}\right)$ to a final state with momenta $\left(k_{1}, k_{2}, k_{3}\right)$ is

$$
\frac{M_{1} M_{2}}{\Delta E+i \Gamma / 2}
$$

where $\Delta E=E\left(p_{1}\right)+E\left(p_{2}\right)-E\left(k_{1}\right)-E_{R}\left(p_{1}+p_{2}-k_{1}\right)$ and $E(k)=\left(m^{2}+k^{2}\right)^{1 / 2}$. The $\Gamma$ in the propagator is the width of the intermediate resonance in the medium. The rate for the reaction is given by the modulus squared of the amplitude (1) summed over final states 
and multiplied by the initial nucleon densities,

$$
\begin{aligned}
W= & n_{1} n_{2} n_{3} \int \frac{d^{3} k_{1}}{(2 \pi)^{3}} \frac{d^{3} k_{2}}{(2 \pi)^{3}} \frac{d^{3} k_{3}}{(2 \pi)^{3}}\left|M_{1}\right|^{2} \frac{1}{(\Delta E)^{2}+\Gamma^{2} / 4}\left|M_{2}\right|^{2} \\
& \times(2 \pi)^{4} \delta^{(4)}\left(k_{1}+k_{2}+k_{3}-p_{1}-p_{2}-p_{3}\right) .
\end{aligned}
$$

It is convenient to write this in the factorized form,

$$
\begin{aligned}
W= & n_{1} n_{2} \int \frac{d^{3} k_{1}}{(2 \pi)^{3}} \frac{d^{4} k_{R}}{(2 \pi)^{4}}\left|M_{1}\right|^{2} \frac{\Gamma}{(\Delta E)^{2}+\Gamma^{2} / 4}(2 \pi)^{4} \delta^{(4)}\left(k_{1}+k_{R}-p_{1}-p_{2}\right) \\
& \times \frac{1}{\Gamma} n_{3} \int \frac{d^{3} k_{2}}{(2 \pi)^{3}} \frac{d^{3} k_{3}}{(2 \pi)^{3}}\left|M_{1}\right|^{2}(2 \pi)^{4} \delta^{(4)}\left(k_{2}+k_{3}-k_{R}-p_{3}\right) \\
= & \int d^{4} k_{R} \frac{d^{4} W_{N N \rightarrow N R}}{d k_{R}^{4}} \frac{\Gamma_{N R \rightarrow N N^{*}}}{\Gamma},
\end{aligned}
$$

where we have replaced the $\delta^{(4)}$-function in (2) by a convolution of two functions. In the last line we have expressed the rate as the product of a rate for producing a generally offshell particle, $W_{N N \rightarrow N R}$, times the branching ratio for that particle to produce $N^{*}$ before disappearing from the system, $\Gamma_{N R \rightarrow N^{*}} / \Gamma$. Beyond this, $\Gamma^{-1} d^{4} W_{N N \rightarrow N R} / d k_{R}^{4}$ in (3) represents a contribution of the $N N$ interactions to the number of off-shell particles $R$ per unit phase-space and energy at any instant. We will return to this interpretation in the conclusion.

When a delta is produced in the intermediate state of the reaction and propagates on shell, the integral for the rate is dominated by the region of the integrand with $\Delta E \sim \Gamma$ due to the factor $\left((\Delta E)^{2}+\Gamma^{2} / 4\right)^{-1}$. Assuming that all other factors in the integrand are constant over this range, the integral over $k_{R}^{0}$ can be evaluated using

$$
\int \frac{d k_{R}^{0}}{2 \pi} \frac{\Gamma}{\left(k_{R}^{0}-E_{R}\left(k_{r}\right)\right)^{2}+\Gamma^{2} / 4}=1
$$

The result is

$$
\begin{aligned}
W_{\Delta}= & n_{1} n_{2} \int \frac{d^{3} k_{1}}{(2 \pi)^{3}} \frac{d^{3} k_{\Delta}}{(2 \pi)^{3}}\left|M_{1}\right|^{2}(2 \pi)^{4} \delta^{(4)}\left(k_{1}+k_{\Delta}-p_{1}-p_{2}\right) \\
& \times \frac{1}{\Gamma_{\Delta}} n_{3} \int \frac{d^{3} k_{2}}{(2 \pi)^{3}} \frac{d^{3} k_{3}}{(2 \pi)^{3}}\left|M_{2}\right|^{2}(2 \pi)^{4} \delta^{(4)}\left(k_{\Delta}+p_{3}-k_{2}-k_{3}\right) \\
= & \int d \Omega_{N \Delta} \frac{d W_{N N \rightarrow N \Delta}}{d \Omega_{N \Delta}} \frac{\Gamma_{N \Delta \rightarrow N N^{*}}}{\Gamma_{\Delta}} .
\end{aligned}
$$


Here $W_{N N \rightarrow N \Delta}$ is the physical rate for producing on-shell deltas in the medium.

When the off-shell propagation dominates, we can neglect $\Gamma$ in the denominator in Eq. (2). The integration over momenta in Eq. (2) is conveniently done by going first to the cm frame of particles 2' and 3' in the final state, which allows an angular integration to be done trivially. The integral that remains to be evaluated for the off-shell rate is then

$$
W_{o f f}=n_{1} n_{2} n_{3} \frac{4 \pi}{(2 \pi)^{5}} \int_{0}^{k_{\max }} d k_{1} \int d \Omega_{k_{1}} k_{1}^{2} k_{2} \mu_{k_{2}} \frac{\left|M_{1} M_{2}\right|^{2}}{(\Delta E)^{2}} .
$$

Here $\mu_{k_{2}}=\left(1 / \sqrt{m_{N}^{2}+k_{2}^{2}}+1 / \sqrt{m_{N^{*}}^{2}+k_{2}^{2}}\right)^{-1}$ is the reduced mass associated with $k_{2}, k_{1}$ is the momentum in 3 -body $\mathrm{cm}$ frame, and the limit $k_{\max }$ is determined by the threshold where $k_{2}=0$.

The interesting quantity for our purposes is the ratio of the rates by the two mechanisms. We reduce Eq. (5) in the similar way we derived Eq. (6), obtaining

$$
W_{\Delta}=\frac{n_{1} n_{2} n_{3}}{\Gamma_{\Delta}} \frac{4 \pi}{(2 \pi)^{4}} \int d \Omega_{k_{1}} k_{1} \mu_{k_{1}} k_{2} \mu_{k_{2}}\left|M_{1} M_{2}\right|^{2} .
$$

Here $\overrightarrow{k_{1}}$ is in the $N \Delta \mathrm{cm}$ frame. Assuming now that the variation of elements $M_{1,2}$ is small on the scale of the integrations, the ratio of the two rates becomes

$$
\frac{W_{o f f}}{W_{\Delta}}=\frac{\Gamma_{\Delta}}{2 \pi} \frac{\int_{0}^{k_{\max }} d k_{1} d \Omega_{k_{2}} k_{1}^{2} k_{2} \mu_{k_{2}} /(\Delta E)^{2}}{\int d \Omega_{k_{1}} k_{1} \mu_{k_{1}} k_{2} \mu_{k_{2}}} .
$$

The r.h.s. of Eq. (8) depends only on $\Gamma$ and kinematic quantities; the dependence on matrix elements is canceled out by our assumption that they are the same for nucleons as for deltas.

We are now ready to evaluate numerically the relative contribution of the two mechanisms. We choose an initial beam momentum of $p_{b}=1.9 \mathrm{GeV} / \mathrm{c}$, which is below the two-body threshold at $2.1 \mathrm{GeV} / \mathrm{c}$, but above both three-body thresholds. We take the mass and width of the delta to be $m_{\Delta}=1.23 \mathrm{GeV}$ and $\Gamma_{\Delta}=0.12 \mathrm{GeV}$. First, let us consider first the situation where particle 1 is from the beam and the other two are in the target, i.e. $\left(p_{1}, p_{2}, p_{3}\right)=\left(p_{b}, 0,0\right)$. For the delta mechanism, the first scattering (between 1 and 2) produces a delta whose momentum in the $N \Delta \mathrm{cm}$ system is $k_{\Delta}=0.5 \mathrm{GeV} / \mathrm{c}$. There is enough energy to produce an $\mathrm{N}^{*}(1535)$ only if the delta goes forward; the solid angle integration in Eq. (17) extends only over $\Omega_{\Delta} \lesssim 0.2 \times 4 \pi$. The approximate magnitudes of the 
other quantities in this angular range are $k_{2} \approx 0.2 \mathrm{GeV} / \mathrm{c}$ and $\mu \sim m_{N} / 2$. For the off-shell mechanism, there is no kinematic restriction on the intermediate state and the full range of solid angle contributes. The range of the $k_{1}$ integration is determined by the available energy to produce the $\mathrm{N}^{*}(1535)$; for our conditions $k_{\max } \approx 0.4 \mathrm{GeV} / \mathrm{c}$ in the 3 -body $\mathrm{cm}$ frame. The remaining quantity to include is $\Delta E \approx-0.5 \mathrm{GeV} / \mathrm{c}$. One can estimate the integrals with these numbers to get a ratio $W_{\text {off }} / W_{\Delta} \approx 15 \%$.

From this result the on-shell theory looks reasonably accurate, but we still have to examine other combinations of projectile and target particle in the basic graph in Fig. 1. It is sufficiently general to consider one particle from the projectile and two from the target, but we need also to consider the case where 1 and 2 are in the same nucleus, i.e. $\left(p_{1}, p_{2}, p_{3}\right)=$ $\left(0,0, p_{b}\right)$. Now the first interaction represents an initial-state correlation. It obviously cannot make an on-shell delta, but it can give a nucleon the momentum needed to produce a heavy particle in the second interaction. The energy denominator is small in the kinematic region where $\overrightarrow{k_{1}}$ is maximal and in the forward direction, reaching $\Delta E=-0.01 \mathrm{GeV}$ at the limit. However, phase space favors larger values of off-shell energy with phase-space average being $\langle\Delta E\rangle \approx-0.1 \mathrm{GeV}$. The ratio of integrals with these numbers is $W_{\text {corr }} / W_{\Delta} \approx 3$.

Thus we find that the mechanism involving initial state correlations will dominate the threshold production of heavy particles, at least early in a reaction. Under this mechanism, the target correlations cause a target nucleon to acquire a large backward momentum before it interacts with a projectile nucleon. In the example above, the momenta of the nucleon moving backwards in the target frame range from $0.1 \mathrm{GeV} / \mathrm{c}$ to $1 \mathrm{GeV} / \mathrm{c}$. While this nucleon is highly off-shell, overall enough energy is gained in the two-nucleon frame of two nucleons to allow the final heavy-particle production. Generally, because of the presence of energetic nucleons from the other nucleus, the high momenta from correlations can play a role and materialize in interactions. When $p_{1}$ and $p_{2}$ are from the same nucleus, then $\Gamma^{-1} d^{4} W_{N N \rightarrow N N} / d k^{4}$ in (3) represents simply a contribution of $N N$ interactions to the ground-state nucleon spectral-function. When the heavy particle production is to take place deeply below the $N N$ threshold, the correlations involving just two target nucleons may not 
suffice to generate the necessary energy in the three-body $\mathrm{cm}$ frame. It may be then advantageous to involve more nucleons in the initial-state correlation. The view that correlations dominate threshold production has been implicit in calculations of Ref. [8] and the effects of two- and more-particle correlations have been included there. As a heavy-ion reaction progresses, the balance in the rates may shift from the mechanism involving correlations to the mechanism with on-shell particles only.

We conclude with a few remarks on possible inclusion of correlations in BUU calculations. An important concept here is the time associated with the off-shell propagation. In scattering, a time delay $\tau$ may be associated with the propagation of the nucleon given by $\tau=\hbar \Gamma /\left((\Delta E)^{2}+\Gamma^{2} / 4\right)[9,10]$. The $\tau$ in the case we considered is very short, which would allow the effects to be treated as an effective three-body interaction. A phenomenological model to introduce such many particle interactions into calculations has already been described in Ref. [11].

This work was initiated at the Institute for Nuclear Physics. We thank U. Mosel for conversations. Support for the work was given by the National Science Foundation under Grant No. PHY-9403666 and by the Department of Energy under Grant No. DE-FG0690ER40561. 


\section{REFERENCES}

[1] G. F. Bertsch and S. Das Gupta, Phys. Rep. 160 (1988) 189.

[2] G. Batko, W. Cassing, U. Mosel, K. Niita, and Gy. Wolf, Phys. Lett. B 256 (1991) 331;

S. Teis, W. Cassing, T. Maruyama, and U. Mosel, Phys. Rev. C 50 (1994) 388.

[3] Gy. Wolf, W. Cassing, and U. Mosel, Nucl. Phys. A552 (1993) 549; Gy. Wolf, W. Cassing, W. Ehehalt, and U. Mosel, Prog. Part. Nucl. Phys. 31 (1993) 273; U. Mosel, in X. Viñas ed., "Proc. Int. Workshop on the Dynamical Features of Nuclei and Finite Fermi Systems", Sitges, 1993, (World Scientific, Singapore, 1994), p. 222.

[4] G. Q. Li, T. Maruyama, Y. Lofty, S. W. Huang, D. T. Khoa, and A. Faessler, Nucl. Phys. A537 (1992) 631; G. Q. Li, S. W. Huang, T. Maruyama, Y. Lofty, D. T. Khoa, and A. Faessler, ibid. 645; S. W. Huang, G. Q. Li, T. Maruyama, and A. Faessler, ibid. A547 (1992) 653; S. W. Huang, A. Faessler, G. Q. Li, R. K. Puri, E. Lehmann, D. T. Khoa, and M. A. Matin, Phys. Lett. B 298 (1993) 41; G. Q. Li, A. Faessler, and S. W. Huang, Prog. Part. Nucl. Phys. 31 (1993) 159.

[5] C. Hartnack, J. Jaenicke, L. Sehn, H. Stöcker, and J. Aichelin, Nucl. Phys. A580 (1994) 643.

[6] V. Metag, Prog. Part. Nucl. Phys. 30 (1993) 75.

[7] B. A. Li, Phys. Rev. C 50 (1993) 2144; B. A. Li, C. M. Ko, and G. Q. Li, ibid. R2575.

[8] P. Danielewicz, Phys. Rev. C 42 (1990) 1564.

[9] P. Danielewicz and S. Pratt, Phys. Rev. C, in press.

[10] A. Messiah, Quantum Mechanics (John Wiley, New York, 1958), p. 400.

[11] G. Batko, J. Randrup, and T. Vetter, Nucl. Phys. A536 (1992) 786; ibid. A546 (1992) 761. 


\section{FIGURES}

FIG. 1. The graph for heavy particle production below the two-body threshold. 


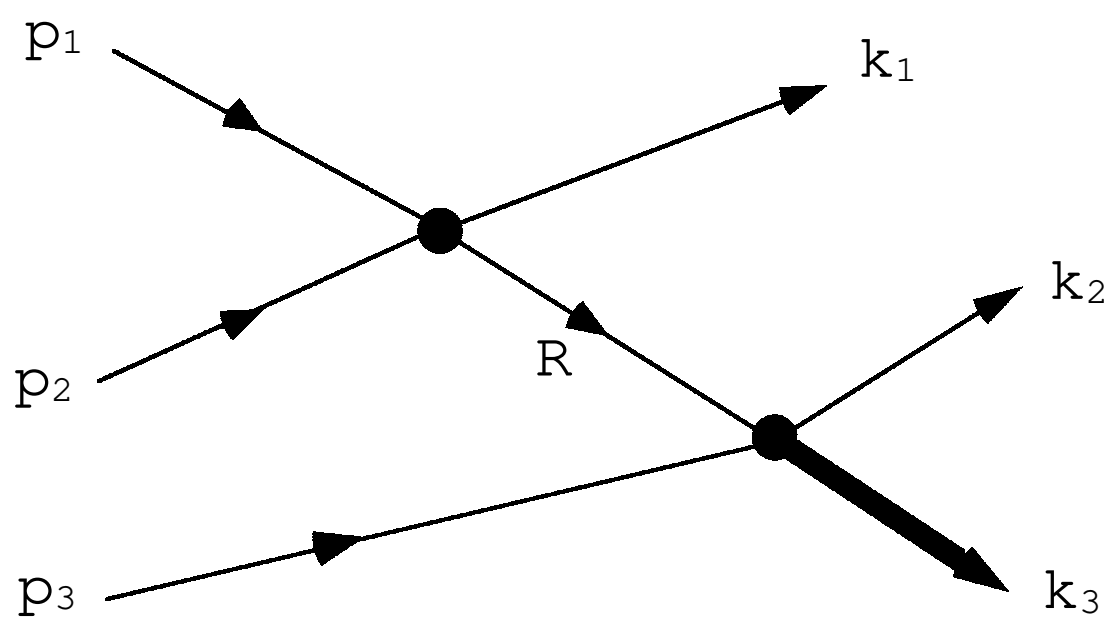

